\title{
Model of Integrated Pool/Conventional/Alternative Electricity Market Operation using Pay-as-Bid pricing
}

\author{
Fabio Stacke ${ }^{1}$ and Pablo Cuervo ${ }^{2}$ \\ ${ }^{1}$ Brazilian Electricity Regulatory Agency - ANEEL \\ Brasília, Brazil \\ SGAN 603 CEP 70.830-030 \\ Phone/Fax number:+55 61 96457997/+556121928944, e-mail: fabiostacke@aneel.gov.br \\ ${ }^{2}$ University of Brasília \\ Brasília, Brazil \\ Dep. Engenharia Elétrica -ENE, Asa Norte, CEP 70.910-900 \\ Phone/Fax number:+55 $6132735977 /+556132746651$, e-mail: pablo@ene.unb.br
}

\begin{abstract}
A pricing model considering the simultaneous interaction of pool, conventional and alternative markets in a power system is presented. This work inserts a competition for alternative power plants separating the pricing for these three markets. The model use the 'Pay-as-Bid' (PAB) pricing approach in opposite of classical marginal pricing (MP) approach because the inelastic demand characteristic and the necessity of reduce total operation cost. In the model pricing approach, an integration process involves an AC Optimal Power Flow (OPF) for obtaining awarded bids in the pool and market with the presence of conventional or alternative energy bilateral contracts. As a result, the obtained prices of pool, conventional and alternative energy incorporate the influences of the topology, voltage levels, losses and capacity limits of generators and transmission lines. Results show that agents can plan their portfolios based on reasonable stable prices that reflect the impact of supplying several electricity types and associated costs of resources in several operation scenarios and bid strategies. From the perspective of the system regulator, the minimization of payments by $\mathrm{PAB}$ ensures the supply of energy, transmission losses and alternative energy requirements as well as enforcing financial adequacy. Numerical cases are presented for evaluating the model.
\end{abstract}

\section{Key words}

Alternative Power Plants, Pay as Bid, Pool, Conventional and Alternative Energy Markets, Average Price.

\section{Introduction}

In recent years, electricity markets have developed important economic and operational tools looking for efficiency in terms of determining prices that represent the costs of production and transmission of energy. From the structural point of view, there are many forms of markets auctions for trading energy [1] but because of different cost of production, is need to separate conventional and alternative markets. The possible advantage of this auction structure is that the voluntary participation of traders in markets could provide efficiency, like in a pool, avoiding the use of complex optimization tools. In the market auctions, electricity market products (conven- tional and alternative energy) are procured simultaneously through central auctions. The advantage of this integrated market is that the resulting prices better reflect the cost of resources due to the inherent relationship between conventional and alternative energy.

From the theoretical point of view, two approaches are mainly followed for pricing electricity: One of them is the classical marginal pricing (MP) where nodal prices represent the cost of the last MW to be supplied and the other is the "Pay-as-Bid" pricing (PAB) which is the way forward bilateral contracts are negotiated. Whether one or the other should be followed has been object of controversy as discussed in [6]. Recently, PAB pricing has showed an increasing interest because some markets are essentially based on bilateral agreements with agents motivated to minimize the volatility of nodal prices and the system regulator is also interested in obtaining financial adequacy $[4,5]$.

This paper analyzes the characteristics of a pricing model designed for working under the classical Marginal Price (MP) and the Pay as Bid (PAB) strategies in a combined market structure involving the presence of long term conventional and alternative energy bilateral contracts and short term trades like pool market. The purpose of the model is exploiting the advantages of centralized market coordination and the potential benefits of using the $\mathrm{PAB}$ pricing strategy with the main objective that is insert alternative power plants in energy system. It is not the focus here to discuss which of the pricing approaches should be followed but instead observing their behavior in the combined market.

The alternative energy market is composed of several types of generators. The characterization and requirements of these services are previously defined by the regulator and are based on the quality of their generation. The PAB version of this model is implemented through an integration process based on the Auman-Shapley [3,5] technique using an AC- OPF that takes into consideration the non-linear characteristic of the transmission network and allows unbundling the use and prices of several services.

The model characteristics allow market agents to plan their portfolios by knowing how prices of electricity 
interact in several possible operation scenarios. From the ISO point of view, it is possible to estimate the impact of different levels and distributions of total bilateral trades and pool load while enforcing price stability and financial adequacy.

The paper is organized as follows. Section 3 presents the formulation. Section 4 describes the pricing mechanism. Section 5 shows how revenues and payments are obtained. Section 6 describes the how to obtain generators and loads portfolios. Section 7 shows the reconciliation of costs. Section 8 shows numerical examples and Section 9 presents conclusions.

\section{Nomenclature}

For each generator at bus $i$ and demand at bus $j$ in the integrated market we define the following notation.

\section{Generators Bid Functions in $(\$ / h)$ :}

$$
\begin{array}{ll}
C_{g i}\left(p_{g i}^{p}\right) & \text { Pool Energy } \\
C_{g i}^{C V}\left(p_{g i}^{C V}\right) & \text { Conventional Energy } \\
C_{g i}^{A L n}\left(p_{g i}^{A L n}\right) & \text { Alternative Energy type } n
\end{array}
$$

Variables:

$p_{g i}^{p} \quad$ Pool Active power generation awarded level (MW)

$q_{g i} \quad$ Reactive power generation level (Mvar)

\begin{tabular}{|c|c|}
\hline $\begin{array}{l}C V \\
g i\end{array}$ & ive power generation level \\
\hline $\begin{array}{l}A L n \\
g i\end{array}$ & $\begin{array}{l}\text { Alternative Active power generation type } n \\
\text { level at bus i (MW) }\end{array}$ \\
\hline di & $\begin{array}{l}\text { Pool Active power demand level at bus } j \\
\text { (MW) }\end{array}$ \\
\hline & $\begin{array}{l}\text { Conventional Active power demand level at } \\
\text { bus } j \text { (MW) }\end{array}$ \\
\hline $\boldsymbol{O}_{d i}^{A L n}$ & $\begin{array}{l}\text { Alternative Active power demand type } n \\
\text { level at bus } j \text { (MW) }\end{array}$ \\
\hline & $\begin{array}{l}\text { Reactive power demand level at bus } j \\
\text { (Mvar) }\end{array}$ \\
\hline $3 C^{C V}$ & $\begin{array}{l}\text { Bilateral Contract for Conventional power } \\
\text { plants }\end{array}$ \\
\hline$C^{A L}$ & $\begin{array}{l}\text { Bilateral Contract for Alternative power } \\
\text { plants type } n\end{array}$ \\
\hline
\end{tabular}

$v_{i}, \delta_{i} \quad$ Module and Angle of Bus $i$ voltage phasor

Constant Parameters:

\section{Formulation}

In this section is presented the formulation of the combined market of energy with pool, conventional and alternative energy bilateral contracts. We consider a one hour auction with no inter-temporal constrains and suppose that enough support of reactive power is available to keep voltages close to nominal values.

\section{A. Pool and Energy Auction}

The combined pool and energy auction determine awarded energy bids for a period of one hour. In this market low cost merit order lists for energy (conventional and alternative) are obtained by minimizing the following objective function.

$$
\text { Minimize } C_{\text {pool }}+C_{\text {conventional }}+C_{\text {alternative }}
$$

Where,

$$
\begin{aligned}
& C_{\text {pool }}=\left\{\sum_{i} C_{i}\left(p_{g i}^{p}\right)\right\} \\
& C_{\text {conventional }}=\left\{\sum_{i} C_{i}^{C V}\left(p_{g i}^{C V}\right)\right\} \\
& C_{\text {alternative }}=\left\{\sum_{i} C_{i}^{A L 1}\left(p_{g i}^{A L 1}\right)+\sum_{i} C_{i}^{A L 2}\left(p_{g i}^{A L 2}\right)\right. \\
& \left.+\ldots+\sum_{i} C_{i}^{A L n}\left(p_{g i}^{A L n}\right)\right\}
\end{aligned}
$$

Cost bid $^{1}$ functions can be considered as continuous quadratic or piece-wise linear functions.

\section{B. Transmission Network}

From the Independent System Operator - ISO point of view, awarded bids and firm bilateral contracts must attend operation constraints imposed by the transmission system. Therefore, feasible solutions should belong to the set defined by constraints (2) to (7) for all buses $i$ and where long term physical bilateral contracts and the pool demand are known quantities. Each demand $j$ is considered with follow energy components $p_{d j}=p_{d j}^{p}+p_{d j}^{C V}+p_{d j}^{A L 1}+\ldots+p_{d j}^{A L n} \quad, \quad$ and generator at bus $i$ also has follow energy components $p_{g i}=p_{g i}^{p}+p_{g i}^{C V}+p_{g i}^{A L 1}+\ldots+p_{g i}^{A L n}$. The network load flow equations are represented by (2) and (3). Constraint (4) represents transmission line limit of active flow, (5) to (7) represent generation capacity limits and voltage limits. Vector $V$ represents bus voltage modules and vector $\delta$ represents bus voltage phase angles. Both vectors have dimension equal to the total number of buses $z$.

$$
\begin{gathered}
p_{g i}-p_{d i}=p_{i}(\mathrm{v}, \delta), \quad \rightarrow \lambda_{i} \\
q_{g i}-q_{d i}=q_{i}(\mathrm{v}, \delta), \quad \rightarrow \lambda_{i}^{q} \\
-p_{i j}^{\max } \leq p_{i j} \leq p_{i j}^{\max } \\
p_{g i}^{\min } \leq p_{g i} \leq p_{g i}^{\max } \\
q_{g i}^{\min } \leq q_{g i} \leq q_{g i}^{\max } \\
v_{i}^{\min } \leq v_{i} \leq v_{i}^{\max }
\end{gathered}
$$

Lambdas in (2) and (3) are Lagrange multipliers associated to each constraint and represent nodal prices for active and reactive power.

\section{Alternative Market Characteristics}

Generators agents can bid in $n$ kinds of alternative plant generator, as in (1). The type of generation defines the quality of each energy. Cleaner services are considered of higher quality than the others. In order to open

\footnotetext{
${ }^{1}$ Under pay-as-bid, generators bid above their true cost. In this paper, generator cost is understood to mean bid cost.
} 
possibilities for participation in conventional market, it is also allowed the possibility to substitute one eventual higher conventional bid. But the substitution consists in allowing that services with better quality and lower cost can substitute services with lower quality and higher cost $[1,7]$. Hence, a feasible bid selection in the energy market auction should belong to the set described by constraints (8) to (13).

$$
\begin{gathered}
B C^{C V} \leq \sum_{i=1}^{n} p_{g i}^{C V} \rightarrow \lambda^{C V} \\
B C^{A L 1} \leq \sum_{i=1}^{n} p_{g i}^{A L 1} \rightarrow \lambda^{A L 1} \\
B C^{A L 1}+B C^{A L 2} \leq \sum_{i=1}^{n} p_{g i}^{A L 1}+\sum_{i=1}^{n} p_{g i}^{A L 2} \rightarrow \lambda^{A L 2(10)} \\
B C^{A L 1}+B C^{A L 2}+B C^{A L n} \leq \\
\sum_{i=1}^{n} p_{g i}^{A L 1}+\sum_{i=1}^{n} p_{g i}^{A L 2}+\sum_{i=1}^{n} p_{g i}^{A L n} \rightarrow \lambda^{A l n} \\
p_{g i}^{C V} \geq 0, p_{g i}^{A L 1} \geq 0, p_{g i}^{A L 2} \geq 0, \ldots, p_{g i}^{A L n} \geq 0 \\
p_{g i}^{C V} \leq p_{g i}^{C V \max }, p_{g i}^{A L 1} \leq p_{g i}^{A L 1 \max }, p_{g i}^{A L 2} \leq p_{g i}^{A L 2 \max } \\
\ldots, p_{g i}^{A L n} \leq p_{g i}^{A L n \max }
\end{gathered}
$$

Where, the estimated required system amounts of each type of energy are considered known and defined by the ISO before the market auction is performed. Lambda variables are Lagrange multipliers associated to each constraint. Upper limits in (13) are related to physical limits such as generators ramp rates and they are part of the information of the alternative energy bids.

\section{Long Term Bilateral Contracts}

Private long term bilateral contracts are considered as firm physical (not financial) contracts which are authorized and implemented by the ISO taking into consideration the reliability conditions of the transmission network. In a compact form, bilateral contracts can be grouped in a GD matrix where each coefficient $G D_{i j}$ represents the MW traded between generators at bus $i$ and load at bus $j$. Therefore, the total amount of contracts supplied by generator $i$ is,

$$
p_{g i}=\sum_{j=1}^{n} G D_{i j}
$$

In addition, the total amount of contracts supplying the demand at bus $j$ is,

$$
p_{d j}=\sum_{i=1}^{n} G D_{i j}
$$

In this model is supposed that firm long-term bilateral contracts already exist at the moment of performing one auction [4]. Because of this, the already committed capacity with bilateral contracts imposes a constraint on the lower generation limit for generators participating in the pool market as shown in (16).

$$
\begin{aligned}
& \qquad p_{g i} \leq p_{g i}^{\max } \\
& \text { E. Alternative Energy Availability of Generators } \\
& \text { Capacity }
\end{aligned}
$$

In the combined market, besides attending bilateral and pool loads, each generator $i$ can also participate by bidding in several alternative energy markets based on its availability and quality. The alternative energy bids should respect the operational capacity limits and generation quality of each generator as described in (17) to (19).

$$
\begin{array}{r}
p_{g i}^{p}+p_{g i}^{C V}+p_{g i}^{A L 1}+p_{g i}^{A L 2}+\ldots+p_{g i}^{A L n} \\
\leq p_{g i}^{\max A L n}(\text { more quality) } \\
p_{g i}^{p}+p_{g i}^{C V}+p_{g i}^{A L 1} \leq p_{g i}^{\max A L 1}(\text { less quality) } \\
p_{g i}^{p}+p_{g i}^{C V} \leq p_{g i}^{\max C V}(\text { conventional) }
\end{array}
$$

\section{F. Marginal Price of Energy}

The Lagrangean function of the optimization problem described in (1) to (19) allows to obtain expressions for the market clearing prices of energy based on the Lagrange Multipliers which represent the sensitivity of cost in terms of energy quality requirements as shown in (20) to (23).

$$
\begin{gathered}
\frac{\partial L}{\partial B C^{A L n}}=\lambda^{A L 1}+\lambda^{A L 2}+. .+\lambda^{A L n}=M C P_{A L n} \\
\frac{\partial L}{\partial B C^{A l 2}}=\lambda^{A L 1}+\lambda^{A L 2}=M C P_{A L 2} \\
\frac{\partial L}{\partial B C^{A l 1}}=\lambda^{A L 1}=M C P_{A L 1} \\
\frac{\partial L}{\partial B C^{C V}}=\lambda^{C V}=M C P_{C V}
\end{gathered}
$$

Since Lagrange multipliers are positive, this formulation ensures not reversal prices among services. In other words, $M C P_{A L n} \geq M C P_{A l 2} \geq M C P_{A L l}$ as discussed in [1].

\section{Pay-as-Bid Incremental Model}

The pay as bid version of this model is implemented through an integration process based on the AumanShapley technique $[3,5]$ that takes into consideration the non-linear characteristic existing in the transmission network due to transmission losses and voltage behaviors. The integration process allows also to unbundling the use and prices of several services including pool dispatch, conventional and alternative energy bilateral contracts. One iteration of the process is described as follows.

Step 1: Bilateral Contracts Losses and Congestion

In this step, losses attributed to bilateral contracts due to the use of the transmission network are compensated in the Pool market. Initially, bilateral load is incremented by $d G D_{i j}$ while holding fixed the pool load and energy 
quality requirements whose value at the very beginning are nil. In this step, the optimization problem minimizes costs of loss compensation due to bilateral contracts and possible congestion management. More precisely, the incremental optimization problem to be solved is defined by (1) to (23) considering for each load $j$ an incremental variation $d p_{d j}=\sum_{i} d G D_{i j}$ while holding fixed pool loads $p_{d j}^{p}$ and energy quality requirements $p_{d j}^{C V}$, $p_{d j}^{A L 1}, p_{d j}^{A L 2}, \ldots, p_{d j}^{A L n}$. Calling the solution of this problem as $d p_{g i}^{*}$ then the contract incremental losses and congestion management are obtained through (24).

$$
d p_{g i}^{\text {losses }}=d p_{g i}^{*}-\sum_{j=1}^{n} d G D_{i j}
$$

Step 2: Pool and Energy Market

In this step, the incremental optimization problem (1) to (24) is solved incrementing pool loads $j$ by $d p_{d j}^{p}$. Energy quality requirements are incremented consecutively by $d p_{d j}^{C V}, d p_{d j}^{A L 1}, d p_{d j}^{A L 2}, \ldots, d p_{d j}^{A L n}$ while holding fixed the bilateral contract loads. The solution of this optimization problem gives the awarded pool incremental generation level,$d p_{g}^{p}$, and energy incremental generation levels, $d p_{g j}^{C V}, d p_{g j}^{A L 1}, d p_{g j}^{A L 2}$ $, \ldots, d p_{g j}^{A L n}$.

\section{Integration Process}

The integration process consists in performing alternatively step 1 and step 2 for small load increments following a uniform integration path from zero to the final value of the load, according to the " $t$ " parameter such that $0 \leq t \leq 1$, as shown in (25).

$$
X=\int_{t=0}^{1} d x(t)
$$

Vector $d x$ is composed of variables $d p_{g i}^{p} d p_{g j}^{C V}$, $d p_{g j}^{A L 1}, d p_{g j}^{A L 2}, \ldots, d p_{g j}^{A L n}$.

\section{Revenues and Payments}

Economic indexes are presented incrementally in this section. Their corresponding final values are obtained by performing the previously described integration process.

Bilateral Contracts

Because bilateral contracts are negotiated in private, their prices are not available. Nevertheless, we adopt as a price for these contracts the corresponding incremental bilateral costs of generators i.e., $I C_{g i}=d C_{g i}\left(p_{g i}\right) / d P_{g i}$.

Revenues: The generator $i$ revenue due to only bilateral contracts is,

$$
d c_{g i}=I C_{g i} \cdot \sum_{j=1}^{n} d G D_{i j}
$$

Payments: Load $j$ pays the supplied bilateral contracts according to (27),

$$
d c_{d j}=\sum_{i=1}^{n} I C_{i} \cdot d G D_{i j}
$$

Bilateral contracts should pay for losses and congestion management according to (28),

$$
d c_{i j}^{\text {losses }}=\sum_{i=1}^{n}\left(\lambda_{j}-\lambda_{i}\right) \cdot d G D_{i j}
$$

This amount could be split among contract parties in a $50 / 50$ basis or other proportion; we adopt a split of 50/50 among contract parties. The corresponding payment of generator $i$ for all its bilateral contracts losses and congestion is,

$$
d c_{g i}^{\text {losses }}=(1 / 2) \sum_{j=1}^{n} d c_{i j}^{\text {losses }}
$$

Similarly, the payment of the bilateral load $j$ is,

Pool and Energy

$$
d c_{d j}^{\text {losses }}=(1 / 2) \sum_{i=1}^{n} d c_{i j}^{\text {losses }}
$$

Revenues: Generator $i$ has a revenue portfolio from participating in attending pool demand, bilateral loss compensation and congestion management as well as providing availability of energy market (conventional and alternative) given by (31) to (34).

$$
\begin{gathered}
d \hat{c}_{g i}^{p}=\lambda_{i} \cdot d p_{g i}^{p} \\
d c_{g i}^{\text {losses }}=\lambda_{i} \cdot d p_{g i}^{\text {losses }} \\
d c_{g i}^{C V}=M C P_{C V} \cdot d g_{g i}^{C V} \\
d c_{g i}^{A L n}=M C P_{A L n} \cdot d g_{g i}^{A L n}
\end{gathered}
$$

Incremental revenues from other alternative energy follow the same calculations.

Payments: Load $j$ has a payment portfolio due to the use of pool demand including losses and energy market following (35). In the case of energy market, load $j$ pays in a pro-rate manner as shown in (36) and (37).

$$
\begin{gathered}
d \hat{c}_{d j}^{p}=\lambda_{j} d p_{d j}^{p} \\
d c_{d j}^{C V}=M C P_{C V} \cdot\left(\sum_{i=1}^{n} d g_{g i}^{C V}\right) \cdot\left(p_{d j}^{C V}\right) / p_{d \text { Total }}^{C V} \\
d c_{d j}^{A \ln }=M C P_{A \ln } \cdot\left(\sum_{i=1}^{n} d g_{g i}^{A L n}\right) \cdot\left(p_{d j}^{A L n}\right) / p_{d \text { Total }}^{A L n}
\end{gathered}
$$

Incremental load payments related to the other services are obtained in a similar way.

\section{Generators and Loads Portfolios}

At the end of the integration process by using (25), we have the net portfolio revenue of generator $i$ which is composed basically by three terms corresponding to pool and energy markets (conventional and alternative) as follows,

$$
c_{g i}=c_{g i}^{p}+c_{g i}^{C V}+c_{g i}^{A L}
$$

Where,

$$
\begin{gathered}
c_{g i}^{A L}=c_{g i}^{A L 1}+c_{g i}^{A l 2}+\ldots+c_{g i}^{A L n} \\
c_{g i}^{p}=\hat{c}_{g i}^{p}-c_{g i}^{\text {losses }}
\end{gathered}
$$


Likewise, the portfolio payment of load $j$ is composed by three terms corresponding to pool and energy markets (conventional and alternative) as follows,

Where,

$$
c_{d j}=c_{d j}^{p}+c_{d j}^{C V}+c_{d j}^{A L}
$$

$$
\begin{gathered}
c_{d i}^{A L}=c_{d i}^{A L 1}+c_{d i}^{A l 2}+\ldots+c_{d i}^{A L n} \\
c_{d j}^{p}=\hat{c}_{d j}^{p}+c_{d j}^{\text {losses }}
\end{gathered}
$$

\section{Reconciliation of Costs}

Under the PAB scheme, the costs allocated to the loads and bilateral contracts perfectly match the generation cost components [5]. This characteristic is also verified in the alternative market at the end of the integration process by applying (25). For instance, in the case of the convencional and alternative energy we have,

$$
\begin{aligned}
& \sum_{i=1}^{n} c_{g i}^{C V}=\sum_{j=1}^{n} c_{d j}^{C V} \\
& \sum_{i=1}^{n} c_{g i}^{A L n}=\sum_{j=1}^{n} c_{d j}^{A L n}
\end{aligned}
$$

\section{Numerical Results}

This section analyzes results of an IEEE 5-Bus system when the suggested pricing model is applied. Table 1 shows the network data in per unit in a base of 100 MVA and $200 \mathrm{kV}$. For simplicity, it is considered enough reactive support to keep bus voltages within limits. Table 2 describes generator bids with capacity limits and continuous bid functions $c_{g i}$. The only two alternative energy market required by the system are $p_{d j}^{A L 1}=14.4$ MW and $p_{d j}^{A L 2}=40 \mathrm{MW}$ whose sum represent a margin of $5 \%$ of total load (i.,e., $54.4 \mathrm{MW}$ ). In order to government incentive the amount of data, the generators bid functions for alternative energy type 2 are considered double of the corresponding conventional energy bids and for alternative energy type 1 are considered triple in Table 2 (i.e., $a_{g 1}^{A L 1}=60 \quad \$ / \mathrm{MWh} \quad$ and $b_{g 1}^{A L 1}=0.12 \$ / \mathrm{MW}^{2} \mathrm{~h}$ for the generator 1 alternative energy bid, $\quad a_{g 1}^{A L 2}=40 \quad \$ / \mathrm{MWh} \quad$ and $b_{g 1}^{A L 2}=0.08 \$ / \mathrm{MW}^{2} \mathrm{~h}$ for the generator 2 alternative energy bid and similar for the other generators alternative energy bids). Alternative energy bids for all generators are considered more expensive and equal to the corresponding conventional energy bids. In order to simplify the observation, cases with transmission congestion and the influence of ramp constraints are not presented. The total fixed system load of $1088 \mathrm{MW}$ is distributed among buses according with the following vector: $p_{d}=[34,85,119,323,527]^{T}(\mathrm{MW})$. This load is supplied by bilateral and pool markets in a proportion of $94 \%$ of bilateral contracts and $6 \%$ of pool load. Table 3 shows the corresponding bilateral contracts matrix, GD.
In this case, the pool loads required at each bus are the coefficients of vector $p_{d}^{p}=[2,5,7,19,31]^{T}(\mathrm{MW})$. Bilateral tariffs are chosen by, $\pi_{i j}^{C V}=d C_{g i}\left(P_{g i}^{C V}\right) / d P_{g i}$ for all bilateral loads $j$.

TABLE I. - Network Data

\begin{tabular}{|c|c|c|c|c|c|c|c|}
\hline \multirow{2}{*}{\multicolumn{2}{|c|}{ Bus }} & \multicolumn{6}{|c|}{ Loads (MW) } \\
\hline & & 1 & 2 & 3 & 4 & 5 & Total \\
\hline \multirow{6}{*}{$\sum_{\substack{0 \\
0}}^{\substack{\pi \\
0}}$} & 1 & 32 & 48 & 32 & 144 & 160 & 416 \\
\hline & 2 & 0 & 32 & 32 & 112 & 240 & 416 \\
\hline & 3 & 0 & 0 & 48 & 48 & 96 & 192 \\
\hline & 4 & 0 & 0 & 0 & 0 & 0 & 0 \\
\hline & 5 & 0 & 0 & 0 & 0 & 0 & 0 \\
\hline & Total & 32 & 80 & 112 & 304 & 496 & \\
\hline
\end{tabular}

\begin{tabular}{|c|c|c|c|c|c|}
\hline $\begin{array}{c}\text { From } \\
\text { Bus }\end{array}$ & $\begin{array}{c}\text { To } \\
\text { Bus }\end{array}$ & $\begin{array}{c}r \\
(\mathrm{pu})\end{array}$ & $\begin{array}{c}x \\
(\mathrm{pu})\end{array}$ & $\begin{array}{c}b \\
(\mathrm{pu})\end{array}$ & $\begin{array}{c}p_{\text {flow }}^{\max } \\
(\mathrm{pu})\end{array}$ \\
\hline 1 & 2 & 0.0147 & 0.168 & 0.138 & 3.00 \\
\hline 1 & 4 & 0.0108 & 0.126 & 0.102 & 3.55 \\
\hline 2 & 3 & 0.0185 & 0.210 & 0.185 & 3.00 \\
\hline 3 & 4 & 0.0294 & 0.336 & 0.296 & 3.00 \\
\hline 3 & 5 & 0.0221 & 0.252 & 0.213 & 3.00 \\
\hline 4 & 5 & 0.0108 & 0.126 & 0.104 & 4.50 \\
\hline 2 & 4 & 0.0105 & 0.130 & 0.100 & 3.60 \\
\hline
\end{tabular}

TABLE II. - Generators Conventional Energy Bids

$C_{g i}\left(p_{g i}\right)=c_{0 i}+a_{i} \cdot p_{g i}+0.5 \cdot b_{i} \cdot p_{g i}^{2}(\$ / h)$
\begin{tabular}{|c|c|c|c|c|c|}
\hline Bus & $\begin{array}{l}p_{g}^{\min } \\
(M W)\end{array}$ & $\begin{array}{l}p_{g}^{\max } \\
(M W)\end{array}$ & $\begin{array}{c}c_{0} \\
(\$ / \mathrm{h})\end{array}$ & $\begin{array}{c}a \\
(\$ / \mathrm{MWh})\end{array}$ & $\begin{array}{c}b \\
(\$ / \mathrm{MW} 2 \mathrm{~h})\end{array}$ \\
\hline 1 & 0 & 460 & 0 & 20 & 0.040 \\
\hline 2 & 0 & 500 & 0 & 21 & 0.030 \\
\hline 3 & 0 & 500 & 0 & 25 & 0.045 \\
\hline 4 & 0 & 500 & 0 & 56 & 0.040 \\
\hline 5 & 0 & 500 & 0 & 57 & 0.040 \\
\hline
\end{tabular}

TABLE III. - Bilateral Contracts (MW)

The case is presented in Table 4. The first six rows illustrate the three market dispatches. Generators 1 and 2 are heavily committed with bilateral contracts $(83 \%$ of their capacity) imposing an out of merit operation for pool market. The economic dispatch only awards pool bids of generator 2 and 3 for attending pool load and transmission losses of $43.63 \mathrm{MW}$. Only the low cost generator 2 participates in the three markets. Due to the low cost of the alternative energy type 1 of generators, the alternative energy type 2 bids are not accepted. Rows 10 to 15 show economic indexes such as nodal prices, incremental costs and average prices of services of the three markets.

TABLE IV. - Conventional and Alternative Energy market dispacht through the integrated $\mathrm{PAB}$ approach with revenues and payments. Bilateral Contracts participation is equal to $94 \%$ of total load. Power in MW, $\lambda, \pi_{g}^{C V}$ and average cost in $\$ / \mathrm{MWh}$. Rows with * contain given data.

\begin{tabular}{|c|c|c|c|c|c|c|}
\hline Bus & 1 & 2 & 3 & 4 & 5 & Total \\
\hline \hline$P_{g}^{C V}$ & 416.0 & 416.0 & 192.0 & 0.0 & 0.0 & 1024.0 \\
\hline$P_{g}^{p}$ & 0.0 & 47.8 & 59.8 & 0.0 & 0.0 & 107.6 \\
\hline$P_{g}$ & 416.0 & 463.8 & 251.8 & 0.0 & 0.0 & 1131.6 \\
\hline \hline
\end{tabular}




\begin{tabular}{|c|c|c|c|c|c|c|}
\hline$P_{g}^{A L 1}$ & 37.6 & 16.8 & 0.0 & 0.0 & 0.0 & 54.4 \\
\hline$P_{g}^{A L 2}$ & 0.0 & 0.0 & 0.0 & 0.0 & 0.0 & 0.0 \\
\hline $\begin{array}{l}\text { Total } \\
P_{d}^{A L}\end{array}$ & 37.6 & 16.8 & 0.0 & 0.0 & 0.0 & 54.4 \\
\hline$* P_{d}^{C V}$ & 32.0 & 80.0 & 112.0 & 304.0 & 496.0 & 1024.0 \\
\hline$* P_{d}^{p}$ & 2.0 & 5.0 & 7.0 & 19.0 & 31.0 & 64.0 \\
\hline$* P_{d}^{A L}$ & 34.0 & 85.0 & 119.0 & 323.0 & 527.0 & 1088.0 \\
\hline$\lambda$ & 34.5 & 34.9 & 36.3 & 37.1 & 39.9 & - \\
\hline$I C_{g}$ & 36.6 & 34.9 & 36.3 & 56.0 & 57.0 & - \\
\hline$* \pi_{g}^{C V}$ & 36.6 & 33.5 & 33.6 & 56.0 & 57.0 & - \\
\hline$C_{g}^{E V} / P_{g}^{C^{V}}$ & 28.3 & 27.2 & 29.3 & - & - & - \\
\hline$C_{g}^{p} / P_{g}^{p}$ & - & 21.7 & 26.3 & - & - & - \\
\hline$C_{g}^{A L} / P_{g}^{4 L}$ & 41.5 & 42.5 & - & - & - & - \\
\hline$c_{g}^{C V}$ & 11781 & 11331 & 5629.4 & 0.0 & 0.0 & 28742 \\
\hline$c_{g}^{p}$ & 0.0 & 1636.0 & 2091.9 & 0.0 & 0.0 & 3727.9 \\
\hline$c_{g}^{A L}$ & 1560.6 & 714.1 & 0.0 & 0.0 & 0.0 & 2274.6 \\
\hline $\begin{array}{l}\text { Total } \\
\text { Rev. } \\
\end{array}$ & 13341 & 13681 & 7721.3 & 0.0 & 0.0 & 34744 \\
\hline$c_{d}^{C V}$ & 898.2 & 2245.5 & 3143.7 & 8532.9 & 13922 & 28742 \\
\hline$c_{d}^{p}$ & 116.5 & 291.2 & 407.7 & 1106.7 & 1805.7 & 3727.9 \\
\hline$c_{d}^{A L}$ & 71.1 & 177.7 & 248.8 & 675.3 & 1101.8 & 2274.6 \\
\hline $\begin{array}{l}\text { Total } \\
\text { Pay. }\end{array}$ & 1085.8 & 2714.4 & 3800.2 & 10314 & 16829 & 34744 \\
\hline
\end{tabular}

Due to the fact that the bilateral contracts only exist for the more economical generators, their average bilateral prices are lower than their corresponding incremental costs. Average prices for energy are lower than marginal prices with average prices for alternative energy being slightly lower than the corresponding marginal clearing price, $\mathrm{MCP}_{\mathrm{AL} 2}$, which is equal to $43.0 \$ / \mathrm{MWh}$. \%). If the same generator bids were used in a marginal pricing approach, the total cost of operation is $42,050.8 \$ / \mathrm{h}$ which represents an increase of $21.0 \%$ in relation to the total cost of $34,744.9 \$ / \mathrm{h}$ obtained by the PAB approach.

\section{Conclusion}

Numerical cases show that even considering alternative energy bids independent of the conventional energy bids in a joint market, the resulting energy prices depend on the committed capacity allocated to supply the long term bilateral contracts and pool demand and associated prices. This interaction represents economic signals for both pool, conventional and alternative energy markets allowing generators to be able to estimate and plan their opportunity cost and portfolios in a consistent way because of the stability of prices and adequacy obtained in the three markets by using PAB. The more stable and adequate behavior (if compared with the nodal prices under marginal pricing) is verified by observing different firm bilateral contract distributions and represents an advantage for market purposes. Moreover, the pricing model always allows obtaining the reconciliation of revenue and payment portfolios in several operation conditions. In the numerical case presented the integration error is not greater than 0.001 and this precision is obtained within a number of 17 integration steps. It is also worth to mention that the classical marginal pricing approach is obtained in this formulation when only one integration step is performed.

In this paper a pricing model is presented with the following characteristics: i) Incorporation of bilateral, pool, conventional and alternative energy markets in a joint market of energy; ii) The combined market allows assessing the impact of electricity products interactions on the operation and consequently on prices; iii) Allows to compare the pay-as-bid pricing and uniform pricing approaches in several scenarios; iv) Market agents can obtain detailed portfolios in terms of revenues and payments for awarded bids; v) ensures the reconciliation between payments and revenues with a reasonable price stability; vi) Allows to obtain economic signals for estimating opportunity costs of electricity products; vii) Allows the possibility of testing several operating scenarios with price strategies in order to evaluate the impact on agents portfolios; and viii) Incentive participation of alternative power plants in energy market.

Further research is under development for studying in detail the impact of fault uncertainty, inter-temporal constraints and the model simplification for application on large power systems.

\section{References}

[1] Alayawan, Ziad, Papalexopoulos, Alex D., Rothleder, Mark e Wu, Tong. "Pricing Energy and Ancillary Services in Integrated Market Systems by an Optimal Power Flow", IEEE Trans. Power Systems, July 2002.

[2] Arroyo, J.M, Galiana, F.D. "Energy and Reserve Pricing in Security and Network-Constrained Electricity Markets", IEEE Trans. Power Systems, vol., 20, No.2, May 2005.

[3] Bakirtzis, A., “Auman-Shapley Congestion Pricing”, Letters in IEEE Power Engineering Review, Vol.21, Issue 3, March 2001, pp.67-69.

[4] F.D. Galiana, I. Kockar, P. Cuervo, "Combined Pool/Bilateral dispatch. I. Performance of Mixed Trading Strategies", IEEE Trans. Power Syst., vol. 17, no.1, pp. 92-99, February 2002.

[5] Cuervo, P., Kockar, I., Galiana, F., "Combined Pool/Bilateral Operation: Part III - Unbundling Costs of Trading Services", IEEE Trans. Power Systems, vol.17, November, p.1191-1198, 2002.

[6] Ren,Y., Galiana, F., "Pay-as-Bid versus Marginal Pricing Part I: Strategic Generator Offers", IEEE Trans. Power Systems, vol.19., No.4, November, 2004. 